http://dx.doi.org/ 10.22319/rmcp.v9i4.4546

Nota de investigación

\title{
Frecuencia de Neospora caninum en bovinos doble propósito en fincas del estado Guárico, Venezuela
}

Frequency of Neospora caninum in double purpose cattle on herds at the State of Guárico, Venezuela

Juan Carlos Pinilla León ${ }^{\text {ab * }}$

Natalia Da Silva Borges ${ }^{b}$

${ }^{a}$ Universidad de Santander. Facultad de Ciencias Exactas, Naturales y Agropecuarias, Programa de Medicina Veterinaria, Campus de Bucaramanga, Lagos de Cacique, Bucaramanga, Santander, Colombia.

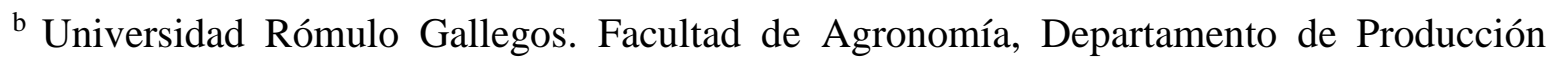
Animal. San Juan de Los Morros, Venezuela.

* Autor de correspondencia: j.pinilla@mail.udes.edu.co

\section{- Resumen:}

Se condujo una investigación con el propósito de determinar la frecuencia de Neospora caninum en bovinos de doble propósito. Se colectaron 890 muestras de sangre distribuidas en cuatro grupos etarios: 0-6 meses, 6-12 meses, 12-24 meses y > 24 meses. La presencia de anticuerpos se determinó mediante un kit de ELISA indirecto, y los resultados se clasificaron en positivos y negativos. La seroprevalencia general fue de $21 \%$ en bovinos, y $76.6 \%$ en las fincas evaluadas. Al referir los resultados al grupo etario no se encontró asociación estadística $(P>0.05)$; sin embargo, el riesgo de ser seropositivo incrementa con la edad, indicando la alta probabilidad de transmisión horizontal de $N$. caninum en el rebaño. Igualmente no hubo asociación estadística con respecto al sexo $(P>0.05)$. Con relación al estatus reproductivo, se encontró asociación estadística $(P<0.05)$ con respecto al aborto. Aunque todos los grupos resultaron positivos, las vacas con historia de aborto mostraron mayor seroconversión, lo que 
indica que el riesgo de aborto aumenta con niveles crecientes de anticuerpos contra el parásito. Del total de fincas positivas (76.6), se observó mayor seroconversión (100) en las que tenían mayor cantidad de perros ( $>7$ perros), y este resultado indica que a mayor cantidad de perros, mayor prevalencia de $N$. caninum, ya que los perros son la principal fuente de transmisión horizontal. Se concluye que existe circulación antigénica de $N$. caninum asociada a abortos en bovinos del estado Guarico, y que pudiera ser controlada mejorando las condiciones sanitarias de las explotaciones.

- Palabras clave: Explotaciones, Ganado vacuno, Parásitos, Prevalencia, Protozoarios.

\section{- Abstract:}

It was carried out an investigation to determine the frequency of Neospora caninum in double purpose cattle. Blood samples $(n=890)$ were collected in four age groups: 0-6 mo, 6-12 mo, 12-24 mo and >24 mo. The presence of antibodies was determined by an indirect ELISA kit, and the results were classified as positive and negative. The seroprevalence was $21 \%$ in cattle, and $76.6 \%$ in the herds evaluated. Regarding to the age group, no statistical association was found $(P>0.05)$; however, the risk of being seropositive increased with age, indicating the high probability of horizontal transmission of $N$. caninum in the herd. There was no statistical association with respect to sex $(P>0.05)$. Regarding reproductive status, a statistical association $(P<0.05)$ was found with respect to abortion. Although all the groups were positive, cows with a history of abortion showed higher seroconversion, indicating that the risk of abortion increased with increasing levels of antibodies against the parasite. From the total positive farms $(76.6 \%)$, higher seroconversion $(100 \%)$ were observed in those with the highest number of dogs ( $>7 \mathrm{dogs})$, and this result indicates that a higher number of dogs, a higher prevalence of $N$. Caninum, since dogs are the main source of horizontal transmission. It is concluded that there is antigenic circulation of N. caninum associated to abortions in double purpose cattle of the State of Guarico, and that could be controlled improving the sanitary conditions of the herds.

- Key words: Cattle, Explotations, Parasites, Prevalence, Protozoans.

Recibido 03/07/2017

Aceptado 23/01/2018 
La Neosporosis es una enfermedad parasitaria causada por un Apicomplexa denominado Neospora caninum, protozoo intracelular obligado que ocasiona aborto en bovinos ${ }^{(1)}$. El ciclo biológico es indirecto, señalando al perro como hospedero definitivo ${ }^{(1)}$; sin embargo, los coyotes, lobos y zorros también han sido identificados como hospederos definitivos ${ }^{(2)}$. Como hospederos intermediarios se han señalado a bovinos, ovinos, caprinos, equinos, venados, perro, otros mamíferos e inclusive las aves de $\operatorname{corral}^{(3)}$. El impacto económico de la neosporosis bovina se debe a que el aborto es el principal signo clínico, ya que las vacas infectadas pueden presentar abortos sucesivos con un alto riesgo de transmitir en forma vertical la infección a su descendencia, por lo cual son consideradas no aptas para la reproducción, lo que amerita el reemplazo de animales de alto valor genético y zootécnico por vientres sanos negativos a este parásito ${ }^{(4,5)}$. Diversos estudios sobre neosporosis bovina, han demostrado la presencia del parásito en las fincas y su estrecha relación con la presencia de abortos, causando grandes pérdidas económicas ${ }^{(6,7)}$. Los abortos frecuentes se convierten en el principal problema de las ganaderías presentándose de forma esporádica, endémica o epidémica con origen infeccioso o no infeccioso, por lo que a veces resulta difícil determinar el agente causal ${ }^{(8)}$.

La neosporosis bovina ha sido señalada en Europa, África, Australia, Nueva Zelanda y América ${ }^{(9)}$. Los estudios serológicos sobre neosporosis bovina en Venezuela demuestran que la enfermedad está distribuida en varios estados y regiones ganaderas, con niveles variables de frecuencia serológica, afectando varios tipos de razas ${ }^{(10,11,12)}$. En los estados Yaracuy y Lara se reportó 17.09 y $44 \%$ de frecuencia en bovinos doble propósito y leche, respectivamente ${ }^{(13,11)}$, y alta ocurrencia de abortos en vacas lecheras ${ }^{(11)}$. En el año 2012, se determinó $17.23 \%$ de seropositividad por medio de la técnica de Elisa en ganado doble propósito del estado Lara, y no se encontraron diferencias significativas entre la frecuencia serológica con respecto al sexo y al grupo etario, sugiriendo que en los rebaños estudiados, las infecciones por $N$. caninum son predominantemente transmitidas por vía transplacentaria ${ }^{(14)}$. Por su parte, en el estado Falcón se encontró $20.6 \%$ de frecuencia serológica en fincas de doble propósito ${ }^{(15)}$.

En Venezuela, específicamente en el estado Guárico, no existe información científica sobre el comportamiento epidemiológico de este protozoario. Por lo tanto, y en virtud de su alto impacto enconómico sobre la ganadería bovina, este trabajo de investigación se realizó con la finalidad de determinar la frecuencia serológica de Neospora caninum en bovinos doble propósito en fincas ganaderas ubicadas en el estado Guárico, Venezuela.

El estudio se realizó en la localidad de San José de Tiznados, municipio Ortíz del estado Guárico $\left(9^{\circ} 26^{\prime} 00^{\prime \prime} \mathrm{N} 67^{\circ} 33^{\prime} 00^{\prime \prime} \mathrm{O}\right)$. La ganadería y producción de cereales son la principal actividad económica de la región. Las características climatológicas de la zona son de tipo tropical lluvioso de sabana, con estación seca y altas temperaturas. San José de Tiznados se encuentra ubicado a $200 \mathrm{msnm}$, temperatura media anual de $28^{\circ} \mathrm{C}$, la precipitación anual de 1,000 a 1,500 mm, observándose una marcada distribución de las lluvias en dos periodos, 
uno seco que se acentúa entre los meses de noviembre a abril y uno lluvioso que va de mayo a octubre. La vegetación está representada en el bosque seco tropical ${ }^{(16)}$.

Se diseñó un muestreo aleatorio, de tipo descriptivo y corte transversal. Para el estudio se seleccionaron 30 fincas de doble propósito de manera aleatoria y sistemática, según censo de vacunación contra la fiebre aftosa del departamento de epidemiología del Instituto Nacional de Seguridad Agrícola Integral ${ }^{(17)}$.

Para el momento del estudio en ninguna de las fincas se habían aplicado vacunas contra $N$. caninum. El cálculo del tamaño de la muestra se hizo empleando la fórmula del muestreo aleatorio simple ${ }^{(18)}$, para lo cual se estableció un margen de error del $20 \%$ de la prevalencia, un nivel de confianza del $95 \%$ y una prevalencia esperada del $15 \%$ en base a estudios previos realizados en Venezuela ${ }^{(15,20)}$, obteniéndose un tamaño de muestra de 544 animales, de la siguiente manera: $\mathrm{n}=\mathrm{Z}^{2} * \mathrm{p} * \mathrm{q} / \mathrm{e}^{2}$, entonces $\mathrm{n}=1,96 * 15 * 85 /(20 * 15 / 100)^{2}=544$ animales. Sin embargo, el tamaño de la muestra se llevó hasta 890 animales, ya que se contaba con suficientes recursos para el momento de la investigación. La selección de los animales en cada finca se hizo mediante muestreo aleatorio simple y de manera proporcional a la cantidad de animales existentes en cada explotación, y se estratificaron en cuatro grupos de edades ${ }^{(14)}$ : becerros/as ( $0-6$ meses), mautes/as (6-12 meses), novillos/as (12-24 meses) y adultos (>24 meses). Para el análisis de la frecuencia se consideró el sexo, estatus reproductivo y la presencia de perros en las fincas.

Las muestras de sangre se tomaron directamente de la vena yugular, utilizando tubos Vacutainer ${ }^{\circledR} \sin$ anticoagulante, y se transportaron en cavas refrigeradas hasta el laboratorio, para su procesamiento. Todas las muestras se centrifugaron a 7,000 rpm por 5 min para la obtención de suero. Se determinó la presencia de anticuerpos anti-Neospora caninum mediante un kit comercial para el ensayo de inmunoabsorción enzimático de ELISA indirecto (INgezim $N$. caninum del laboratorio Ingenasa). El procesamiento de las muestras se realizó en el laboratorio de investigación en Parasitología de la Universidad "Rómulo Gallegos". El procedimiento se realizó de acuerdo a las instrucciones del fabricante.

La frecuencia serológica se determinó dividiendo el total de casos positivos / total de animales evaluados * 100. Los resultados obtenidos se analizaron mediante estadísticos descriptivos y test de Ji-cuadrada para determinar asociaciones estadísticas entre los valores de frecuencia serológica y grupos etarios, sexo y presencia de perros en fincas. Para los cálculos se utilizó el programa estadístico Statistix ver 8.0 $0^{(19)}$.

Se determinó una frecuencia serológica individual de $21 \%$ en el municipio Ortíz del estado Guárico, encontrando animales seropositivos en 23/30 (76.6\%) de las fincas examinadas. Los resultados obtenidos coinciden con lo señalado en otros estudios ${ }^{(10)}$ donde determinaron $14.09 \%$ de seroprevalencia en vacas de 13 estados de Venezuela, así como $11.3 \%$ en vacas procedentes de fincas doble propósito y $86.7 \%$ de fincas seropositivas ${ }^{(20)}$. Igualmente, los 
resultados obtenidos coinciden con lo reportado por León et al ${ }^{(12)}$ quienes señalaron $13 \mathrm{y}$ $17 \%$ en ganaderías doble propósito del estado Guárico y sur del estado Aragua, respectivamente. Igualmente, en otros estudios se demostró $17.09 \%$ de seroprevalencia en bovinos y $74.51 \%$ en las fincas examinadas del estado Yaracuy ${ }^{(13)}$, así como 17.23 y $20.6 \%$ de seropositividad en ganado doble propósito de los estados Lara y Falcón, respectivamente ${ }^{(14,15)}$. Los resultados obtenidos en este estudio difieren con lo reportado en México y Paraguay, donde se encontraron valores de seroprevalencia a $N$. caninum de 16 a $59 \%$ y $35.7 \%$, respectivamente, así como $44 \%$ de seropositividad en vacas del estado Lara, Venezuela ${ }^{(11)}$. El estudio serológico sobre neosporosis bovina en el municipio Ortiz del estado Guárico demuestra que la enfermedad está ampliamente distribuida en el municipio y fincas evaluadas, lo cual hace necesario implementar medidas de control y prevención.

En el Cuadro 1 se muestra la comparación entre la frecuencia de muestras analizadas según el grupo etario y sexo de los animales. Al referir los resultados al grupo etario, se encontró $14.3 \%$ de frecuencia en becerros, $17.8 \%$ en mautes, $19.5 \%$ en novillos y $23.5 \%$ en adultos, lo que demuestra que la seropositividad aumentó de manera proporcional con la longevidad de los animales, no existiendo asociación estadísitca entre la frecuencia serológica y la edad de los animales. Esto puede deberse a la infección postnatal de los bovinos con fuentes contaminadas con ooquistes esporulados del protozoario. Estos resultados coinciden con lo reportado por otros ${ }^{(14,15,21)}$, quienes señalaron que el riesgo de ser seropositivo incrementa con la edad, indicando la alta probabilidad de transmisión horizontal de $N$. caninum en el hato. La seroprevalencia ligeramente mayor en animales más viejos sugiere que está produciéndose infección postnatal superpuesta a la transmision congénita.

Cuadro 1: Comparación entre la seroprevalencia a N. caninum según grupo etario y sexo

\begin{tabular}{lcccc}
\hline & $\begin{array}{c}\mathbf{N}^{\circ} \text { animales } \\
\text { muestreados }\end{array}$ & $\begin{array}{c}\mathbf{N}^{\circ} \text { animales } \\
\text { seropositivos }\end{array}$ & $\%$ & $\begin{array}{c}\text { Estadístico } \\
\text { de prueba }\end{array}$ \\
\hline Grupo etario (meses) & & & & \\
$0-6$ (becerros) & 84 & 12 & 14.3 & \\
$6-12$ (mautes) & 95 & 17 & 17.8 & $X^{2}: 4.01$ \\
$12-2$ (novillos) & 235 & 46 & 19.5 & $P=0.26$ \\
$>24$ (adultos) & 476 & 112 & 23.5 & \\
Total & 890 & 187 & 21.0 & \\
Sexo & & & & \\
Hembras & 702 & 148 & 21.1 & $X^{2}: 0.01$ \\
Machos & 188 & 39 & 20.7 & $P=0.9$ \\
Total & 890 & 187 & 21.0 & \\
\hline
\end{tabular}

$P>0.05$. 
Aunque ambos sexos resultaron seropositivos, no se encontró asociación estadística $(P>0,05)$ entre la seroprevalencia a $N$. caninum y el sexo (Cuadro 1). Los resultados obtenidos coinciden con lo reportado por Gharekhani et $a l^{(24)}$ quienes no encontraron diferencias estadísiticas con respecto al sexo. Probablemente, las diferencias hormonales juegan un papel importante en la susceptibilidad del huésped a la infección por $N$. caninum, ya que la excreción de estrógenos aumenta la producción de anticuerpos y los andrógenos suprimen las respuestas inmunes de las células T y B, y por lo tanto, la inmunidad contra la infección en vacas puede disminuir ${ }^{(25)}$. Por otro lado, las hembras representan la fuente de transmision transplacentaria de $N$. caninum a su descendencia, ya que determinan la presencia y persistencia de la infección, así como la característica endémica de la neosporosis bovina, ocurriendo la transmisión de vacas infectadas a hijas e incluso la perpetuidad de la infección en futuras gestaciones ${ }^{(1,5,26)}$.

Se observaron diferentes grados de positividad, con respecto al estatus reproductivo (Cuadro 2). Aunque todos los grupos resultaron seroprevalentes a la infección por el protozoario, las vacas con historia de aborto mostraron mayor seroconversión $(71.1 \%, P<0.05)$. Los resultados obtenidos coinciden con lo demostrado en otros trabajos ${ }^{(6,7)}$ donde señalaron la presencia de $N$. caninum y su relación con abortos en ganado lechero; sin embargo, los resultados difieren con lo reportado por Pulido $e t a l^{(27)}$, quienes no encontraron relación estadística entre la presencia de $N$. caninum y abortos en vacas. A pesar de la alta presencia de anticuerpos contra $N$. caninum en vacas con historia de abortos, es importante señalar que existen otros agentes infecciosos como brucelosis, leptospirosis, virus de la rinotraqueítis infecciosa bovina, diarrea viral bovina, etc, que no fueron considerados en el diagnóstico diferencial del estudio, y que deberían ser incluidas en futuras investigaciones. Las vacas seropositivas son más propensas a abortar que las vacas seronegativas, como ha sido demostrado en muchos estudios, y el riesgo de aborto aumenta con niveles crecientes de anticuerpos específicos de $N$. caninum en animales individuales ${ }^{(1)}$. En nuestro estudio se determinó que el porcentaje de abortos fue 2.4 veces mayor en los individuos seropositivos comparado con los seronegativos (71.1 y $28.9 \%$, respectivamente). Estos resultados coinciden con lo demostrado por otros investigadores ${ }^{(27)}$ quienes señalaron que el porcentaje de abortos fue tres veces mayor en animales seropositivos comparados con los negativos. Diferentes estudios han descrito una asociación significativa entre la presencia de la infección y el aborto, con un riesgo 2 a 3.5 veces superior en vacas seropositivas que en las seronegativas ${ }^{(22,23,29)}$. Las vacas congénitamente infectadas tienen hasta 7.4 veces más probabilidades de abortar que una vaca no infectada, además permanecen infectadas de por vida. A pesar de esto, se ha descrito que el riesgo de abortar parece disminuir en las gestaciones siguientes, sin afectar la fertilidad de la vaca ${ }^{(30)}$. 
Cuadro 2: Comparación entre la seroprevalencia a Neospora caninum según estatus reproductivo de las vacas

\begin{tabular}{lccc}
\hline & $\begin{array}{c}\mathbf{N}^{\circ} \text { animales } \\
\text { muestreados }\end{array}$ & $\begin{array}{c}\mathbf{N}^{\circ} \text { animales } \\
\text { seropositivos }\end{array}$ & $\%$ \\
\hline Vacas vacías & 130 & 26 & 20.0 \\
Vacas preñadas & 251 & 35 & 13.9 \\
Vacas repetidoras & 50 & 19 & 38.0 \\
Vacas con HA & 45 & 32 & 71.1 \\
Total & 476 & 112 & 23.5 \\
\hline \multicolumn{4}{c}{$\mathrm{X}^{2}: 76.15 . P<0.05}$. \\
\end{tabular}

Otras alteraciones reproductivas como las repeticiones de celo también han sido asociadas con la seropositividad a $N$. caninum $^{(31,32)}$; sin embargo, los factores que favorecen la probabilidad de que una vaca seropositiva aborte son por lo general desconocidos ${ }^{(32)}$. La relación del estrés y las prácticas de manejo, condiciones climáticas, coinfecciones, edad, etc; pueden desempeñar un papel importante en la reactivación de infecciones latentes ${ }^{(1)}$. En un estudio encontraron una fuerte asociación entre el nivel de anticuerpos en la vaca y la ocurrencia de lesiones histopatológicas en fetos abortados consistentes con la infección por N. caninum $^{(33)}$.

Durante el presente estudio se observó la presencia de perros en todas las fincas visitadas. La mayor frecuencia serológica en animales de mayor edad (23.5 \%) sugiere que la transmisión horizontal está probablemente contribuyendo a la prevalencia en otros grupos etarios, y que los perros al convivir con el ganado podrían servir como posible fuente de infección por medio de la excreción de ooquistes ${ }^{(34)}$; sin embargo, muestras de perros no fueron examinadas durante este estudio. En el Cuadro 3 se muestra la seroprevalencia a $N$. caninum de acuerdo a la cantidad de perros presentes en las fincas. Se observaron diferentes grados de positividad, lo que refleja que hubo asociación estadística $(P<0.05)$ con respecto al número de perros presentes. Del total de fincas positivas (76.6\%), se observó mayor seroconversión $(100 \%)$ en las que tenían mayor cantidad de perros (> 7 perros), y este resultado indica que a mayor cantidad de perros, mayor seroprevalencia de $N$. caninum. En la mayoría de los estudios epidemiológicos, la presencia de perros de granja ha sido un factor de riesgo para la seropositividad en bovinos ${ }^{(1)}$, ya que los perros son hospederos definitivos y principal fuente de transmission horizontal de $N$. caninum. Los ganaderos han reportado la infección postnatal en aquellas fincas donde los perros defecan en los comederos, ensilaje de maíz o heno, mientras que no hay evidencias de infeccion donde no hay perros. Igualmente, se observó infección postnatal con mayor frecuencia en fincas donde los perros se alimentaron con placenta bovina, descargas uterinas y calostro o leche, a diferencia de los rebaños testigo ${ }^{(35)}$. 
Esto sugiere que estos materiales pueden representar una fuente de infección para los perros, es decir, pueden facilitar que los perros se infecten con $N$. caninum. Otros estudios no encontraron una asociación entre los perros y el aborto en los rebaños bovinos ${ }^{(36,37,38)}$. Sin embargo, debido a que los abortos asociados a $N$. caninum no siempre están vinculados a la transmisión horizontal sino que también ocurren en las vacas infectadas crónicamente, no se puede esperar que haya siempre una asociación positiva entre la presencia o el número de perros y el aborto bovino. Uno de los estudios que identificaron una asociación positiva entre la presencia de perros en las fincas y el aborto asociado a $N$. caninum había analizado selectivamente los factores de riesgo para el aborto epidémico. Debido a que el aborto epidémico, posiblemente es causado por la transmisión horizontal mediada por ooquistes, se espera la identificación de la presencia de potenciales hospederos definitivos, es decir, perros de finca, como un factor de riesgo ${ }^{(1)}$.

Cuadro 3: Comparación entre la seroprevalencia a N. caninum en fincas de doble propósito según la cantidad de perros

\begin{tabular}{lccc}
\hline & $\begin{array}{c}\text { Fincas } \\
\text { muestreadas }\end{array}$ & $\begin{array}{c}\text { Fincas } \\
\text { seropositivas }\end{array}$ & $\%$ \\
\hline$<3$ perros & 5 & 2 & $40.0^{\mathrm{a}}$ \\
4 y 6 perros & 9 & 5 & $55.5^{\mathrm{a}}$ \\
$>7$ perros & 16 & 16 & $100.0^{\mathrm{b}}$ \\
Total & 30 & 23 & 76.6 \\
\hline
\end{tabular}

$\mathrm{X}^{2}: 12.43$. a,b $P<0.05$.

De acuerdo a los resultados obtenidos en este estudio, se determinó una frecuencia serológica individual de $21 \%$ en bovinos doble propósito y $76.6 \%$ en las fincas examinadas, lo que demuestra que la infección está ampliamente distribuida en el municipio Ortíz del estado Guarico, lo cual hace necesario implementar medidas de control y prevención. El incremento de la seropositividad con la edad de los animales, así como la presencia de gran cantidad de perros en las explotaciones, presumen la probabilidad de transmisión horizontal de $N$. caninum en los bovinos y fincas examinadas. Sin embargo, habría que formular modelos experimentales para comprobar científicamente tal aseveración. Aunque el riesgo de aborto estuvo asociado con niveles crecientes de anticuerpos específicos de $N$. caninum en las vacas examinadas $(71.1 \%)$, también se deben considerar otros agentes infecciosos como causantes de aborto en ganado bovino. 


\section{N Agradecimientos V}

Al Programa de Medicina Veterinaria de la Facultad de Ciencias Exactas, Naturales y Agropecuarias de la Universidad de Santander por su valiosa colaboración y apoyo en la ejecución de este trabajo.

\section{- Literatura citada:}

1. Dubey JP, Schares G, Ortega-Mora L. Epidemiology and control of neosporosis and Neospora caninum. Clin Microbiol Rev 2007;20(2):323-367.

2. Gondim L, McAllister M, Pitt W, Zemlicka D. Coyotes (Canis latrans) are definitive hosts of Neospora caninum. Int J Parasitol 2004;34:159-161.

3. Moore D, Odeón A, Venturini M, Campero C. Neosporosis bovina: conceptos generales, inmunidad y perspectivas para la vacunación. Rev Arg Microbiol 2005;37:217-228.

4. Björkman C, Johansson O, Syenlud S, Holmdahl J, Uggla A. Neospora species infection in a herd of dairy cattle. J Am Vet Med Assoc 1996;208(9):144-146.

5. Hietala S, Thurmond M. Postnatal Neospora caninum transmission and transient serologic responses in two dairies. Int J Parasitol 1999;29:1669-1676.

6. Oviedo T, Betancur C, Mestra A, González M, Reza L, Calonge K. Estudio serológico sobre neosporosis en bovinos con problemas reproductivos en Montería, Córdoba, Colombia. Rev MVZ Córdoba 2007;12(1):929-933.

7. López G, Restrepo B, Restrepo M, Lotero M, Murillo V, Chica A, Cano J, Giraldo J. Estudio para evidenciar la presencia de Neospora caninum en bovinos de la hacienda San Pedro en el municipio de Fredonia. Rev MVZ CES 2007;2(1):7-20.

8. Neverauskas CE, Nasir A, Reichel MP. Prevalence and distribution of Neospora caninum in water buffalo (Bubalus bubalis) and cattle in the Northern territory of Australia. Parasitol Int 2015;64(5):392-396. 
9. Dubey JP. Neosporosis in cattle: biology and economic impact. J Am Vet Med Assoc 1999;214:1160-1163.

10. García F. La neosporosis como factor limitante emergente de la eficiencia reproductiva en rebaños bovinos [resumen]. Congreso Nacional de Ciencias Veterinarias. Maracay, Venezuela. 2005:147.

11. Obando C, Bracamonte M, Montoya A, Cadenas. Neospora caninum en un rebaño lechero y su asociación con el aborto. Rev Cien FCV-LUZ 2010;20(3):235-239.

12. León E, Guillén A, Aragort W, García F, Morales G, Pino L, Sandoval E, Balestrini C. Limitantes parasitológicas en rebaños doble propósito del Municipio San José de Guaribe (estado Guárico) y Sur del estado Aragua. En: Espinoza F, Dominguez C, editores. I Simposio Tecnologías Apropiadas para la Ganadería de los Llanos de Venezuela. Valle de la Pascua, Venezuela 2007:177-194.

13. Escalona J, García F, Mosquera O, Vargas F, Corro A. Factores de riesgo asociados a la prevalencia de Neosporosis bovina en el municipio Bolívar del estado Yaracuy, Venezuela. Zoot Trop 2010;28(2):201-211.

14. Suárez C, Maldonado J. Seropositividad a Neospora caninum en unidades de producción bovina del estado Lara, Venezuela. Zoot Trop 2012;30(1):35-41.

15. Fernández JG, García F. Diagnóstico serológico de neosporosis bovina en fincas de la región de Tucacas, estado Falcón, Venezuela. Zoot Trop 2013;31(4):291-298.

16. Ministerio del Ambiente y Recursos Naturales Renovables (MARNR). Anuario climatológico. MARNR. Estado Guarico. 2014.

17. INSAI. Instituto Nacional de Seguridad Agrícola Integral. Censo de Vacunación contra la Fiebre Aftosa. Departamento de Epidemiología. 2015.

18. OPS. Organización Panamericana de la Salud. Nota Técnica No. 18. Procedimientos para estudios de prevalencia en enfermedades crónicas por muestreo. Buenos Aires 1979.

19. Statistix 8. Analytical Software for Windows. USA. 2008.

20. Lista-Alves D, Palomares-Naveda R, García F, Obando C, Arrieta D, Hoet A. Serological evidence of Neospora caninum in dual-purpose cattle herds in Venezuela. Vet Parasitol 2006;136:347-349.

21. Bartels C, Arnaiz J, Ruiz A, Björkman C, Frössling J, Von Blumröder D. Supranational comparison of Neospora caninum seroprevalences in cattle in Germany, the Netherlands, Spain and Sweden. Vet Parasitol 2006;137:17-27. 
22. Waldner C, Janzen E, Henderson J, Haines D. Outbreak of abortion associated with Neospora caninum infection in a beef herd. JAVMA 1999;215:1485-1490.

23. Davison H, Otter A, Trees A. Significance of Neospora caninum in British diary cattle determined by estimation of seroprevalence in normally calving cattle and aborting cattle. Int J Parasitol 1999;29(8):1189-1194.

24. Gharekhani J, Esmaeilnejad B, Rezaei H, Yakhchali M, Heidari H, Azhari M. Prevalence of anti-Neospora caninum antibodies in Iranian goats. Annals Parasitol 2016;62(2):111-114.

25. Gharekhani J, Heidari H. Serology based comprehensive study of Neospora infection in domestic animals in Hamedan province, Iran. J Ad Vet An Res 2014;1:119-124.

26. Hall C, Reichel M, Ellis J. Neospora abortions in dairy cattle: diagnosis, mode of transmission and control. Vet Parasitol 2005;128:231-241.

27. Pulido M, Díaz A, Andrade R. Asociación entre variables reproductivas y anticuerpos anti Neospora caninum en bovinos lecheros de un municipio de Colombia. Rev Mex Cien Pecu 2017;8(2):167-174.

28. Mazuz ML, Fish L, Reznikov D, Wolkomirsky R, Leibovitz B, Savitzky I, Golenser J, Shkap V. Neosporosis in naturally infected pregnant dairy cattle. Vet Parasitol 2014;205(1-2):85-91.

29. Sager H, Fischer I, Furrer K, Strasser M, Waldvogel A, Boerlin P, Audigé L, Gottstein B. A Swiss case-control study to assess Neospora caninum-associated bovine abortions by PCR, histopathology and serology. Vet Parasitol 2001;102(1-2):1-15.

30. López-Gatius F, García-Ispierto I, Santolaria P, Yániz JL, López-Béjar M, Norgareda C, Almería S. Relationship between rainfall and Neospora caninum-associated abortion in two dairy herds in a dry environment. J Vet Med 2005;52:147-15.

31. Sierra R, Esparza L, Parra M, Vasquez Z, Vazquez C. Factores de riesgo asociados a la seroprevalencia de anticuerpos a Neospora caninum en ganado lechero de Aguascalientes, México. Rev Mex Cienc Pecu 2011;2(1):15-24.

32. Bruhn F, Daher D, López E, Barbieri J, Da Rocha C, Guimarães A. Factors associated with seroprevalence of Neospora caninum in dairy cattle in southeastern Brazil. Trop Anim Health Prod 2013;45(5):1093-1098.

33. De Meerschman F, Speybroeck N, Berkvens D, Rettigner C, Focant C, Leclipteux T, Cassart D, Losson B. Fetal infection with Neospora caninum in dairy and beef cattle in Belgium. Theriogenology 2002;58:933-945. 
34. Chaparro J, Olivera M, Ramírez N, Villar D, Fernandez J, Londoño J, Palacio L. Neospora caninum serostatus in dairy cattle of the Northern plains of Antioquia, Colombia. Rev MVZ Córdoba 2016;21(3):5577-5583.

35. Dijkstra T, Barkema H, Eysker M, Hesselink J, Wouda W. Natural transmission routes of Neospora caninum between farm dogs and cattle. Vet Parasitol 2002;105:99-104.

36. Mainar-Jaime R, Thurmond M, Berzal-Herranz B, Hietala S. Seroprevalence of Neospora caninum and abortion in dairy cows in northern Spain. Vet Rec 1999;145:7275.

37. Fischer I, Furrer K, Audige' L, Fritsche A, Giger T, Gottstein B, Sager H. Von der Bedeutung der bovinen Neosporose beim Abortgeschehen in der Schweiz. Schweiz Arch Tierheilkd 2003;145:114-12.

38. Romero J, Perez E, Dolz G, Frankena K. Factors associated with Neospora caninum serostatus in cattle of 20 specialized Costa Rican dairy herds. Prev Vet Med 2002;53:263-273. 